\title{
Sustained Attention Deficits as Markers of Genetic Susceptibility to Schizophrenia
}

\author{
WEI J. CHEN* AND STEPHEN V. FARAONE
}

\begin{abstract}
This article reviews recent evidence regarding the potential of the visual sustained attention deficits as measured by the Continuous Performance Test (CPT) as an endophenotype of the genetic susceptibility to schizophrenia. Findings in community subjects indicate that sustained attention develops during the primary school ages, reaches its maximum around early adolescence, and declines with age after adulthood. The assertion that CPT performance deficits, and especially on the more difficult versions, are reliable and valid genetic susceptibility indicators of schizophrenia is supported by the following results: 1) CPT deficits are present in schizophrenic patients, are particularly associated with negative and disorganized symptoms, and deficits on the more difficult CPT versions are not amenable to neuroleptic treatment; 2) subjects with schizotypal personality features also exhibit CPT deficits, which are specifically associated with the negative factor of schizotypy; 3) a substantial proportion of nonpsychotic relatives of schizophrenic patients (19$34 \%$ ) have CPT deficits, which can also be predicted from their probands' CPT performance. Thus, using a CPT deficits as an endophenotype of schizophrenia would not only provide a valuable measure of genetic risk, but would also greatly enhance our understanding of etiology, and may help identify susceptibility genes for schizophrenia. Am. J. Med. Genet. (Semin. Med. Genet.) 97:52-57, 2000 (c) 2000 Wiley-Liss, Inc.
\end{abstract}

KEY WORDS: continuous performance test; endophenotype; heritability; schizotypy; symptom dimension; vulnerability indicator

\section{INTRODUCTION}

Although previous family, twin, and adoption studies have consistently shown that there is genetic contribution to the risk for schizophrenia, the search for genes that influence the predisposition toward schizophrenia has so far been unsuccessful. One obstacle to gene finding is that we cannot identify carriers of genes in the absence of manifest symptoms. Also, psychiatric diagnoses are likely to be heterogeneous in that not all people with the same diagnosis carry the same susceptibility genes [Faraone et al., 1999]. One possible way to surpass the limitations posed by clinical phenotype is through the identification of neurobiological or neuro-

Grant sponsor: National Health Research Institutes, Taiwan; Grant number: DOH88-HR-825, NHRI-GT-Ex89p825p; Grant sponsor: National Science Council, Taiwan; Grant number: NSC88-2314-B002-247, NSC89-2320-B-002-106; Grant sponsor: National Institute of Health; Grant number: R01MH57934, R01HD37694.

${ }^{*}$ Correspondence to: Wei J. Chen, M.D., Sc.D., Institute of Epidemiology, College of Public Health, National Taiwan University, 1 Jen-Ai Road, Section 1, Taipei 100, Taiwan. behavioral characteristics associated with schizophrenia, or endophenotypes, that may be more closely linked to gene expression [Moldin and Erlenmeyer-Kimling, 1994]. We could then use heritable endophenotypes to identify people who carry schizophrenia genes but do not show symptoms of the disorder. This could lead to increased statistical power when conducting genetic analyses [Faraone et al., 1995].

We review evidence regarding the potential of the visual sustained attention deficits as measured by the Continuous Performance Test (CPT) [Rosvold et al., 1956] as an endophenotype of the genetic susceptibility to schizophrenia. Studies before 1994 have been reviewed thoroughly by Nuechterlein [1991] and Cornblatt and Keilp [1994].

\section{Sustained Attention Deficits Measured by the Continuous Performance Test}

The evolution of various versions of the CPT have been described in more detail elsewhere [Nuechterlein, 1991; Cornblatt and Keilp, 1994]. Common features of the CPT include participants responding to predesignated targets among stimuli that are presented at a rapid fixed rate. The discrimination vigilance task of the CPT may consist of a single stimulus (CPT-X, single character or number as the target) or two successive stimuli (CPT-AX, a character or number preceded by another character or number as the target). The difficulty level of the CPT can be raised further by blurring the stimuli (degraded CPT) or using a relative target, i.e., requiring a response to the second stimulus in any pair of identical stimuli (CPT-Identical Pairs Version or CPTIP). It is worth noting that, compared with the simple CPT-X, the CPT-AX or CPT-IP increases the load on working memory, and the degraded CPT increases the load on early sensory processing. The performance indices of the CPT have evolved from the hit rate or false alarm rates alone to indices derived from signal detection theory. Sensitivity $\left(d^{\prime}\right)$ refers to an individual's ability to discriminate target stimuli from nontarget stimuli, whereas the response criterion $(\ln \beta)$ measures the amount of perceptual evidence that the person requires to decide that a stimulus is a target. Given a person's discrimination ability $\left(d^{\prime}\right)$, if he or she adopts a less 
stringent $\ln \beta$, the hit rate will increase whereas the false alarm rate increases at the same time. Because of its joint consideration of both the hit and false alarm rates, $\mathrm{d}^{\prime}$ is usually the variable of interest in studies on sustained attention. Alternatively, a nonparametric sensitivity index that estimates the area under the curve, $\mathrm{A}^{\prime}$, can be computed when the parametric assumptions of $\mathrm{d}^{\prime}$ can not be assured or the hit and false alarm rates are near perfect performance level. Generally speaking, the reliability of both the hit rate and $\mathrm{d}^{\prime}$ of the CPT are excellent $(>0.70)$, whereas that of $\ln \beta$ is less satisfactory (around 0.5).

\section{Effects of Demographic Features on CPT Performance}

Well-established norms for the CPT are essential for choosing a threshold for defining impaired performance. For adults, however, most previous studies have examined only a limited number of normal subjects. The only normative study of CPT performance in the general population was by Chen et al. [1998a], who examined 345 adults randomly selected from a community using both the undegraded and the 25\% degraded CPT-'1-9'. The results of multiple linear regression showed that older ages were associated with a decreasing hit rate and sensitivity $\left(\mathrm{d}^{\prime}\right)$, whereas higher levels of education were associated with an increasing hit rate and $\mathrm{d}^{\prime}$. Men had higher hit rates and $\mathrm{d}^{\prime}$ than women for the degraded CPT.

For children, Greenberg and Waldman's [1993] study of 775 children 6-16 years of age was ideal for describing CPT normal values. The stimuli used in that study, however, were two visual shapes that were easily discriminated. One was designated as the target. This method is different from the CPT versions used in most previous studies. Recently, Lin et al. [1999] examined the relationships of age and gender with CPT-'1-9' performance among 341 randomly selected school children 6-15 years of age. Multiple regression analyses revealed that the hit rate and sensitivity of both the undegraded and the degraded CPT increased with age, whereas the false alarm rate decreased with age. Boys had higher hit rate and sensitivity on the degraded CPT than girls.

Taken together, the findings indicate that sustained attention develops

\section{Taken together, the findings indicate that sustained attention develops during the primary school ages, reaches its maximum around early adolescence, and declines with age after adulthood.}

during the primary school ages, reaches its maximum around early adolescence, and declines with age after adulthood. These data highlight the necessity of standardization with adjustment for age, education, and gender in assessing CPT performance among relatives of schizophrenic patients [Chen et al., 1998b].

\section{CPT Deficits in \\ Schizophrenic Patients}

The first criterion for CPT deficits to be an endophenotype of schizophrenia is that they should be present and stable in schizophrenic patients. Early crosssectional studies reported that schizophrenic patients exhibited CPT performance deficits whether they were chronically hospitalized or in remission [Nuechterlein, 1991]. What has been less clear is whether CPT deficits in schizophrenic patients are mediating vulnerability indicators (performance becomes poorer during overt symptoms than during remission) or stable vulnerability indicators (not amenable to neuroleptic treatment). Cross-sectional studies have produced conflicting results. Several studies found significant differences in CPT performance between medicated and unmedicated patients but others failed to detect such differences [Nuechterlein, 1991; Cornblatt and Keilp, 1994].

A rigorous examination of the impact of course and neuroleptic treat- ment requires longitudinal studies. Several studies that employed the undegraded CPT-X or degraded CPT-X showed worse CPT performance during active psychotic states compared with periods of remission [Orzack et al., 1967; Spohn et al., 1977; Nuechterlein et al., 1991]. One study failed to detect such differences, probably because of small sample sizes and young age [Erickson et al., 1984]. When a working memory component was added to the task, however, as in the case of CPT-AX [Nuechterlein et al., 1991; Epstein et al., 1996; Finkelstein et al., 1997] or CPT-IP [Cornblatt et al., 1997], CPT performance was stable between psychotic and remitted states.

A randomized, double-blind, clinical trial showed that schizophrenic patients' performance on both the undegraded and degraded CPT-'1-9' did not change significantly from baseline to the end of 12-weeks' antipsychotic treatment with risperidone or haloperidol, despite significant reductions in symptom severity [Liu et al., 2000]. Overall, these findings indicate that the different CPT versions might tap different components of sustained attention: the versions requiring working memory (e.g., the undegraded CPTAX, degraded CPT-AX, and CPT-IP) are stable vulnerability indicators, whereas the other versions (e.g., undegraded or degraded CPT-X) might be mediating vulnerability indicators.

\section{CPT Deficits and Clinical Symptom Dimensions of Schizophrenia}

Among studies specifically examining the relationships between CPT performance indexes and clinical symptom dimensions, one of the most consistent findings is that CPT deficits are associated with negative symptoms [Nuechterlein et al., 1986; Hain et al., 1993; Johnstone and Frith, 1996; Liu et al., 1997; Roitman et al., 1997]. But one study that employed hypothesized rather than empirical negative factors failed to find such an association [Strauss et al., 1993]. Buchanan et al. 
[1997] found that schizophrenic patients with the deficit syndrome showed more impaired performance on the CPT compared with other schizophrenic patients. Many studies also found that poorer performance on the CPT were associated with thought disorder [Nuechterlein et al., 1986; Strauss et al., 1993; Pandurangi et al., 1994; Nelson et al., 1998] or disorganized symptoms [Liu et al., 1997]. Meanwhile, all these studies have found that positive symptoms do not correlate with either $\mathrm{d}^{\prime}$ or $\ln \beta$ on the CPT.

\section{CPT Deficits in Other Psychiatric Disorders}

If CPT deficits are vulnerability indicators of schizophrenia, they should not be present in patients with other psychiatric disorders, unless some susceptibility genes for schizophrenia are also susceptibility genes for other disorders. Previous studies have shown worse CPT performance in schizophrenic patients compared with alcoholic patients [Mussgay and Hertwig, 1990], schizoaffective patients [Walker, 1981], and patients with depressive disorders [Cornblatt et al., 1989]. Two studies, however, suggested that CPT performance of bipolar patients did not significantly differ from that of schizophrenic patients [Rund et al., 1992; Addington and Addington, 1997]. Similarly, Nelson et al. [1998] found no difference in CPT performance between patients with major depression with psychosis and schizophrenia. Thus, whether the CPT deficit in schizophrenic patients and that in patients with other psychoses can be differentiated warrants further investigation.

A related issue in younger populations is the CPT deficits found among children with attention deficit hyperactivity disorder (ADHD). An early study reported that children with ADHD differed from normal controls in having lower $\ln \beta$ on the CPT, whereas offspring of schizophrenic mothers differed from normal controls in having lower d' [Nuechterlein, 1983]. In a meta-analysis of 26 studies, however, children with ADHD were found to perform significantly worse than normal controls on the CPT in terms of both commission and omission errors as well as signal-detection indices [Losier et al., 1996]. How to differentiate children with ADHD from children at increased risk for schizophrenia warrants further investigation.

\section{CPT Deficit and Schizotypy}

Genetic vulnerability to schizophrenia may manifest itself in schizophrenialike personality disorders (e.g., schizotypy) rather than a full syndrome of schizophrenia [Gottesman and Shields, 1982; Kendler et al., 1993]. Thus, CPT deficits should be present in persons with schizotypal personality disorder if it is a vulnerability indicator of schizo-

\section{Genetic vulnerability to} schizophrenia may manifest itself in schizophrenia-like personality disorders (e.g., schizotypy) rather than a full syndrome of schizophrenia. Thus, CPT deficits should be present in persons with schizotypal personality disorder if it is a vulnerability indicator of schizophrenia. Indeed, subjects with a high score on schizotypal scales or a diagnosis of schizotypal personality disorder have been found to have poorer CPT performance as compared to nonschizotypal controls.

phrenia. Indeed, subjects with a high score on schizotypal scales [Lenzenweger et al., 1991; Obiols et al., 1993; Chen et al., 1998a] or a diagnosis of schizotypal personality disorder [Condray and Steinhauer, 1992; Harvey et al., 1996] have been found to have poorer CPT performance as compared to nonschizotypal controls.

Interestingly, the relationship between types of schizotypal symptoms and CPT performance in adults is parallel to that between the types of schizophrenic symptoms and CPT performance. The interpersonal or negative features of schizotypy are associated with lower $\mathrm{d}^{\prime}$ on the degraded CPT [Kendler et al., 1991; Chen et al., 1997]. Symptoms of schizotypal disorganization are associated with lower $\mathrm{d}^{\prime}$ on the undegraded CPT [Chen et al., 1997]. Cognitive-perceptual schizotypal symptoms, however, are not associated with d' [Kendler et al., 1991; Chen et al., 1997].

The relationship between schizotypy and CPT performance has also been examined in adolescents. Among randomly selected adolescents, higher scores on the Perceptual Aberration Scale (PAS) was associated with lower $\mathrm{d}^{\prime}$ scores but no symptom dimensions from the Schizotypal Personality Questionnaire were associated with $\mathrm{d}^{\prime}$ on the CPT [Chen et al., 1997]. Obiols et al. [1997] screened for schizotypal adolescents using CPT performance and found that subjects in the lowest decile of CPT performance had higher PAS score than those having better CPT performance.

\section{CPT Deficits in Relatives of Schizophrenic Patients}

Another criterion for $\mathrm{CPT}$ deficits to be vulnerability markers for schizophrenia is that they should be evident among the non-psychotic relatives of schizophrenic patients. Several issues can be addressed in studies examining CPT performance among such relatives. First, the CPT performance in relatives of schizophrenic patients should be poorer than that in normal controls. Early studies comparing children of schizophrenic mothers to children of normal mothers using the CPT-X or CPT-AX did not find significant differences. In contrast, studies using a more difficult CPT could detect such deficits 
in the children of schizophrenic mothers [Nuechterlein, 1991]. Lately, there have been more studies examining CPT performance among adult nonpsychotic relatives of schizophrenic patients, whom have been found to have deficits on the degraded CPT [Grove et al., 1991] or both the undegraded and degraded CPT [Mirsky et al., 1995; Chen et al., 1998b].

Finding CPT differences between relatives of schizophrenia patients and normal controls alone, however, does

\section{In summary, the} accumulated evidence to date supports the idea that CPT performance deficits, especially on the more difficult versions (with working memory demand plus increased loads on early sensory processing), are reliable and valid genetic susceptibility indicators of schizophrenia. Among these, the most comprehensively studied version of the CPT has been the CPT-AX with or without stimulus degradation.

not necessarily make the measure useful for genetic analysis of schizophrenia. A phenotype that leads to a higher ratio $(\lambda$ index) of prevalence among relatives of patients versus the general population would be more powerful for linkage analysis [Risch, 1990]. In a review of reported rates of the putative spectrum phenotype among first-degree relatives of schizophrenic patients, Faraone et al. [1995] found that the $\lambda s$ of the P300 latency measure, two assessments of schizotypal personality disorder, and two measures of neuromotor impairment were between 11-15, whereas that of a composite attention deficit (including the CPT) was 30.

Using data from 148 nonpsychotic relatives and 345 community adults, Chen et al. [1998b] found that $\lambda$ was $>15$ for the undegraded CPT and $>30$ for the degraded CPT with the choice of 3 standard deviations below the population mean as the cutoff point. Thus, the CPT performance deficit might be more useful than other schizophrenia spectrum definitions in linkage analyses. In that study, the proportion of relatives with deficits in $\mathrm{d}^{\prime}$ ranged from 19\% (undegraded CPT) to 34\% (degraded CPT). These percentages are much higher than the morbidity risk for schizophrenia or schizophrenia-related psychoses (about 10\%) among the relatives of schizophrenic patients [Gottesman and Shields, 1982; Kendler et al., 1993].

The idea that CPT deficits reflect the action of schizophrenia susceptibility genes predicts that schizophrenic probands' having CPT deficits should, compared with other schizophrenic probands, have a higher rate of CPT deficits in relatives. When Chen et al. [1998b] divided schizophrenic probands into CPT-deficit and CPT-nondeficit subgroups, their relatives' CPT performance was consistent with this prediction.

Additional studies show that the CPT is heritable. In normal families, the heritability of $\mathrm{d}^{\prime}$ for the CPT-IP ranged from 0.39-0.49 [Cornblatt et al., 1988]. The heritability of $\mathrm{d}^{\prime}$ was 0.79 in a study of nonpsychotic siblings of schizophrenic patients [Grove et al., 1991], whereas estimates of $0.48-0.62$ (undegraded CPT) and 0.51-0.57 (degraded CPT) were found in a study of non-psychotic parents and schizophrenic offspring [Chen et al., 1998b]. Also if CPT scores measure the genetic predisposition to schizophrenia, then CPT performance should be associated with schizotypal features among relatives. In a study of relatives of schizophrenic probands, Chen et al. [1998b] found that CPT performance correlated with schizotypal symptoms of inter- personal dysfunction and disorganization, but not cognitive-perceptual symptoms.

\section{Future Perspectives and Summary}

Several issues must be addressed before CPT deficits can be fruitfully incorporated into the future genetic dissection of schizophrenia. First, the modes of inheritance of CPT performance in schizophrenic families can be explored through segregation analyses. Second, the relative contribution of genetic factors, shared environmental factors and non-shared environmental factors on CPT performance can be estimated from twin studies. Third, the genetic relationship between schizotypy and CPT performance could be elucidated through bivariate analyses in twins.

In summary, the accumulated evidence to date supports the idea that CPT performance deficits, especially on the more difficult versions (with working memory demand plus increased loads on early sensory processing), are reliable and valid genetic susceptibility indicators of schizophrenia. Among

\section{Using CPT deficits as an endophenotype of schizophrenia would not only provide a valuable measure of genetic risk, but would also greatly enhance our understanding of etiology, and may help identify susceptibility genes for schizophrenia.}

these, the most comprehensively studied version of the CPT has been the CPT-AX with or without stimulus degradation. In support of this assertion are the following points: 1) CPT deficits are present in schizophrenic patients, are particularly associated with negative and disorganized symptoms, and deficits on the more difficult CPT 
versions are not amenable to neuroleptic treatment; 2) subjects with schizotypal personality features also exhibit CPT deficits, that are specifically associated with the negative factor of schizotypy; and 3) a substantial proportion of nonpsychotic relatives of schizophrenic patients (19-34\%) have CPT deficits, that can also be predicted from their probands' CPT performance. Using CPT deficits as an endophenotype of schizophrenia would not only provide a valuable measure of genetic risk, but would also greatly enhance our understanding of etiology, and may help identify susceptibility genes for schizophrenia.

\section{ACKNOWLEDGMENTS}

This work was supported by grants DOH88-HR-825 and NHRI-GTEx89p825p from the National Health Research Institutes, Taiwan; and grants NSC88-2314-B-002-247 and NSC892320-B-002-106 from the National Science Council, Taiwan to Wei J. Chen and National Institute of Health Grants R01MH57934 and R01HD37694 to Stephen V. Faraone.

\section{REFERENCES}

Addington J, Addington D. 1997. Attentional vulnerability indicators in schizophrenia and bipolar disorder. Schizophr Res 23:197204.

Buchanan RW, Strauss ME, Breier A, Kirkpatrick B, Carpenter WT Jr.. 1997. Attentional impairments in deficit and non-deficit forms of schizophrenia. Am J Psychiatry 154:363-370.

Chen WJ, Hsiao CK, Lin CCH. 1997. Schizotypy in community samples: the threefactor structure and correlation with sustained attention. J Abnorm Psychol 106: 649-654.

Chen WJ, Hsiao CK, Hsiao L-L, Hwu H-G. 1998a. Performance of the Continuous Performance Test among community samples. Schizophr Bull 24:163-174.

Chen WJ, Liu SK, Chang C-J, Lien Y-J, Chang Y-H, Hwu H-G. 1998b. Sustained attention deficit and schizotypal personality features in nonpsychotic relatives of schizophrenic patients. Am J Psychiatry 155: 1214-1220.

Condray R, Steinhauer SR. 1992. Schizotypal personality disorder in individuals with and without schizophrenic relatives: similarities and contrasts in neurocognitive and clinical functioning. Schizophr Res 7:33-41.

Cornblatt BA, Risch NJ, Faris G, Friedman D, Erlenmeyer-Kimling L. 1988. The Continuous Performance Test, Identical Pairs
Version: I. New findings about sustained attention in normal families. Psychiatry Res 26:223-238.

Cornblatt BA, Lenzenweger MF, ErlenmeyerKimling L. 1989. The Continuous Performance Test, Identical Pairs Version: II. Contrasting attentional profiles in schizophrenic and depressed patients. Psychiatry Res 29:65-85.

Cornblatt BA, Keilp JG. 1994. Impaired attention, genetics, and the pathophysiology of schizophrenia. Schizophr Bull 20:31-46.

Cornblatt B, Obuchowski M, Schnur B, O’Brien JD. 1997. Attention and clinical symptoms in schizophrenia. Psychiatr Quart 68:343359.

Epstein JI, Keefe RSE, Roitman SL, Harvey PD, Mohs RC. 1996. Impact of neuroleptic medications on continuous performance test measures in schizophrenia. Biol Psychiatry 39:902-905.

Erickson WD, Yellin AM, Hopwood JH, Realmuto GM, Greenberg LM. 1984. The effects of neuroleptics on attention in adolescent schizophrenics. Biol Psychiatry 19: 745-753.

Faraone SV, Kremen WS, Lyons MJ, Pepple JR, Seidman LJ, Tsuang MT. 1995. Diagnostic accuracy and linkage analysis: how useful are schizophrenia spectrum phenotypes? Am J Psychiatry 152:1286-1290.

Faraone, SV, Tsuang D, Tsuang MT. 1999. Genetics of mental disorders: a guide for students, clinicians, and researchers. New York: Guilford.

Finkelstein JRJ, Cannon TD, Gur RE, Gur RC, Moberg P. 1997. Attentional dysfunctions in neuroleptic-naive and neurolepticwithdrawn schizophrenic patients and their siblings. J Abnorm Psychol 106:203-212.

Gottesman II, Shields J. 1982. Schizophrenia: the epigenetic puzzle. Cambridge: Cambridge University Press.

Greenberg LM, Waldman ID. 1993. Developmental normative data on the Test of Variables of Attention (T.O.V.A.). J Child Psychol Psychiatry 34:1019-1030.

Grove WM, Lebow BS, Clementz BA, Cerri A, Medus C, Iacono WG. 1991. Familial prevalence and coaggregation of schizotypal indicators: a multitrait family study. J Abnorm Psychol 100:115-121.

Hain H, Maier W, Klinger T, Franke P. 1993. Positive/negative symptomatology and experimental measures of attention in schizophrenia. Psychopathology 26:62-68.

Harvey PD, Keefe RSE, Mitroupolou V, Dupre R, Roitman SL, Mohs RC, Siever LJ. 1996. Information-processing markers of vulnerability to schizophrenia: performance of patients with schizotypal and nonschizotypal personality disorders. Psychiatry Res 60:49-56

Johnstone EC, Frith CD. 1996. Validation of three dimensions of schizophrenic symptoms in a large unselected sample of patients. Psychol Med 26:669-679.

Kendler KS, Ochs AL, Gorman AM, Hewitt JK, Ross DE, Mirsky AF. 1991. The structure of schizotypy: a pilot multitrait twin study. Psychiatry Res 36:19-36.

Kendler KS, McGuire M, Gruenberg AM, O'Hare A, Spellman M, Walsh D. 1993. The Roscommon Family Study: III. Schizophrenia-related personality disorders in relatives. Arch Gen Psychiatry 50:781788 .

Lenzenweger MF, Cornblatt BA, Putnick M. 1991. Schizotypy and sustained attention. J Abnorm Psychol 100:84-89.

Lin CCH, Hsiao CK, Chen WJ. 1999. Development of sustained attention assessed using the Continuous Performance Test among children 6-15 years of age. J Abnorm Child Psychol 27:403-412.

Liu SK, Hwu H-G, Chen WJ. 1997. Clinical symptom dimensions and deficits on the Continuous Performance Test in schizophrenia. Schizophr Res 25:211-219.

Liu SK, Chen WJ, Chang C-J, Lin H-N. 2000. Effects of atypical neuroleptics on sustained attention deficits in schizophrenia: a trial of risperidone versus haloperidol. Neuropsychopharmacology 22:311-319.

Losier BJ, McGrath PJ, Klein RM. 1996. Error patterns on the Continuous Performance Test in non-medicated and medicated samples of children with and without ADHD: a meta-analytic review. J Child Psychol Psychiatry 37:971-987.

Mirsky AF, Yardley SL, Jones BP, Walsh D, Kendler KS. 1995. Analysis of the attention deficit in schizophrenia: a study of patients and their relatives in Ireland. J Psychiatr Res 29:23-42.

Moldin SO, Erlenmeyer-Kimling L. 1994. Measuring liability to schizophrenia: progress report 1994: editors' introduction. Schizophr Bull 20:25-29.

Mussgay L, Hertwig R. 1990. Signal detection indices in schizophrenics on a visual, auditory and bimodal Continuous Performance Test. Schizophr Res 3:303-310.

Nelson EB, Sax KW, Strakowski SM. 1998. Attentional performance in patients with psychotic and nonpsychotic major depression and schizophrenia. Am J Psychiatry 155: 137-139.

Nuechterlein KH. 1983. Signal detection in vigilance tasks and behavioral attributes among offspring of schizophrenic mothers and among hyperactive children. J Abnorm Psychol 92:4-28.

Nuechterlein KH, Edell ES, Norris M, Dawson ME. 1986. Attentional vulnerability indicators, thought disorder, and negative symptoms. Schizophr Bull 12:408-426.

Nuechterlein KH. 1991. Vigilance in schizophrenia and related disorders. In: Steinhauer SR, Gruzelier JH, Zubin J, editors. Handbook of schizophrenia, vol. 5: neuropsychology, psychophysiology and information processing. Amsterdam: Elsevier. p 397-433.

Nuechterlein KH, Dawson ME, Ventura J, Fogelson D, Gitlin M, Mintz J. 1991. Testing vulnerability models: stability of potential vulnerability indicators across clinical state. In: Häfner H, Gattaz WF, editors. Search for the causes of schizophrenia, vol. II. Berlin: Springer-Verlag. p 177-191.

Obiols JE, García-Domingo M, de Trinchería I, Doménech E. 1993. Psychometric schizotypy and sustained attention in young males. Person Indiv Diff 14:381-384.

Obiols JE, Serrano F, Barrantes N, GarciaMarimon M, Gras S, Bosch E, Caparros B, Carandell F. 1997. Frontal dysfunction and psychosis proneness in CPT-linked vulnerable adolescents. Person Indiv Diff 23:677683 
Orzack MH, Kornetsky C, Freeman H. 1967. The effect of daily carphenazine on attention in the schizophrenic patient. Psychopharmacologia 8:31-38.

Pandurangi AK, Sax KW, Pelonero AL, Goldberg SC. 1994. Sustained attention and positive formal disorder in schizophrenia. Schizophr Res 13:109-116.

Risch N. 1990. Linkage strategies for genetically complex traits, II: the power of affected relative pairs. Am J Hum Genet 46:229241.

Roitman SEL, Keefe RSE, Harvey PD, Siever LJ,
Mohs RC. 1997. Attentional and eye tracking deficits correlate with negative symptoms in schizophrenia. Schizophr Res 26: 139-146

Rosvold HE, Mirsky AF, Sarason I, Bransome ED Jr, Beck LH. 1956. A Continuous Performance Test of brain damage. J Consult Psychol 20:343-350.

Rund BR, Orbeck AL, Landro NI. 1992. Vigilance deficits in schizophrenics and affectively disturbed patients. Acta Psychiatr Scand 86:207-212.

Spohn HE, Lacoursiere RB, Thompson K,
Coyne L. 1977. Phenothiazine effects on psychological and psychophysiological dysfunction in chronic schizophrenics. Arch Gen Psychiatry 34:633-644.

Strauss ME, Buchanan RW, Hale J. 1993. Relations between attentional deficits and clinical symptoms in schizophrenic outpatients. Psychiatry Res 47:205-213.

Walker E. 1981. Attentional and neuromotor functions of schizophrenics, schizoaffectives, and patients with other affective disorders. Arch Gen Psychiatry 38:1355-1358. 